Check for updates

Cite this: Soft Matter, 2019, 15, 2009

Received 19th October 2018, Accepted 28th January 2019 DOI: $10.1039 / \mathrm{c} 8 \mathrm{sm} 02136 \mathrm{~h}$

rsc.li/soft-matter-journal

\section{Human blood platelets contract in perpendicular direction to shear flow $\dagger$}

\begin{abstract}
Jana Hanke, ${ }^{a}$ Christiane Ranke, ${ }^{a}$ Eleonora Perego ${ }^{a}$ and Sarah Köster (D) *ab
In their physiological environment, blood platelets are permanently exposed to shear forces caused by blood flow. Within this surrounding, they generate contractile forces that eventually lead to a compaction of the blood clot. Here, we present a microfluidic chamber that combines hydrogel-based traction force microscopy with a controlled shear environment, and investigate the force fields platelets generate when exposed to shear flow in a spatio-temporally resolved manner. We find that for shear rates between $14 \mathrm{~s}^{-1}$ to $33 \mathrm{~s}^{-1}$, the general contraction behavior in terms of force distribution and magnitude does not differ from no-flow conditions. The main direction of contraction, however, does respond to the externally applied stress. At high shear stress, we observe an angle of about $90^{\circ}$ between flow direction and main contraction axis. We explain this observation by the distribution of the stress acting on the adherent cell: the observed angle provides the most stable situation for the cell experiencing the shear flow, as supported by a finite element method simulation of the stresses along the platelet boundary.
\end{abstract}

\section{Introduction}

Blood platelets are the major cellular players during hemostasis ${ }^{1}$ and originate by budding from megakaryocytes. While they contain cytoskeletal components such as actin, tubulin and myosin, they do not possess a nucleus or microtubuleorganizing center. Their size in the resting state is merely 2 to $5 \mu \mathrm{m}$ (diameter) by $1 \mu \mathrm{m}$ (thickness). ${ }^{1}$ Upon activation, they drastically change their shape from this discoidal to a spherical appearance, by elongating the microtubule loops found underneath the cell membrane. ${ }^{2,3}$ When adhering to a substrate, they start to spread to a flat shape, reaching about 30 to $40 \mu \mathrm{m}^{2}$ in area with a height of $100 \mathrm{~nm}$ or less. ${ }^{4-6}$ The activation is initiated via several pathways such as physical triggers, e.g. shear forces, or chemical cues, such as ADP or thrombin. ${ }^{1}$

During the initial clotting process, platelets attach to and spread on the extra-cellular matrix before contracting the blood clot towards the vessel wall and re-establishing blood flow. Thus, blood clotting occurs under continuous shear flow. The shear rates vary greatly in the human body, from 5 to $10 \mathrm{~s}^{-1}$ in larger veins up to $2000 \mathrm{~s}^{-1}$ in small arteries. ${ }^{7,8}$ Hence, platelets must attach, spread and contract under various conditions.

\footnotetext{
${ }^{a}$ Institute for X-Ray Physics, University of Goettingen, 37077 Göttingen, Germany. E-mail: sarah.koester@phys.uni-goettingen.de

${ }^{b}$ German Center for Cardiovascular Research (DZHK), partner site Göttingen, Germany

$\dagger$ Electronic supplementary information (ESI) available: Details of the experimental procedures. See DOI: 10.1039/c8sm02136h
}

It has been shown in a number of studies that the proteins involved in the clotting process change in dependency of the shear rate within the environment. At the lowest physiological shear rates (on the order of $10 \mathrm{~s}^{-1}$ ), attachment is governed by the platelets' response to fibrinogen, ${ }^{9,10}$ followed by collagen I, ${ }^{10,11}$ and then von Willebrand factor (VWF) for higher shear rates (on the order of $1000 \mathrm{~s}^{-1}$ ). ${ }^{9}$ It was shown that the various matrix networks interact with different membrane proteins. Whereas the adhesion to fibrinogen occurs via the integrin $\alpha \mathrm{IIb} \beta 3,{ }^{11}$ the adhesion to VWF occurs via the glycoprotein GP-Ib-V-IX. ${ }^{12}$ In the case of collagen I, both anchor proteins are involved during the attachment process. ${ }^{10}$ Hence, the shear rate acting on the platelet greatly influences the mechanism of adhesion and aggregation during wound healing.

The contraction of single blood platelets or small clots in no-flow environments has been thoroughly characterized by different techniques, including micropost arrays, ${ }^{13,14}$ atomic force microscopy (AFM) ${ }^{15}$ traction force microscopy (TFM) ${ }^{6,16}$ and, most recently, tension sensors. ${ }^{17}$ The measured forces depend greatly on the method used and can reach some hundreds of nN. Further, it was demonstrated that the contractile forces are transmitted at the edge of the platelet. ${ }^{6,17}$

To study the influence of shear flow on the contractile behavior of cells, these methods for measuring forces have to be combined with controlled flow fields as provided by microfluidics. Technically, this has been realized in different ways, typically by combining a polydimethylsiloxane (PDMS) structure and a microscopy glass cover slip. For force measurements under flow conditions, either a micropost array or an elastic 
hydrogel needs to be inserted into the flow chamber preceding the assembly of the entire chamber. The critical step of bonding the two components, i.e. the PDMS cast and the substratecontaining glass slide, was previously achieved by customized sample holders, ${ }^{18-20}$ adhesives $^{21}$ or plasma oxidizing of the PDMS structure followed by press bonding. ${ }^{22}$ However, the addition of adhesives may change the height of the channel by the thickness of the adhesive layer, which would have negative ramifications because of less controlled flow conditions. Further, only oxidizing the PDMS and press bonding with the glass slide does not covalently bind the chamber together, potentially leading to leakage when using higher flow rates or for prolonged measuring times. To covalently bind the glass slide to the PDMS structure, Lam et al. ${ }^{23}$ chose to plasma oxidize both components, protecting their protein coated micropost array during plasma exposure by a removable piece of PDMS. A similar approach was chosen by Jannat et al., ${ }^{24}$ who plasma oxidized both the PDMS and the hydrogel-containing glass slide. In this case, the hydrogel was not protected during plasma treatment and adhesive proteins were added after assembly to the chamber.

Such combined TFM-microfluidics approaches have led to interesting findings. Endothelial cells, which play an important role during angiogenesis, ${ }^{25}$ were observed to not contract in parallel to the flow as suggested by the previously observed geometrical alignment of the cell. ${ }^{23,26}$ Instead, several studies including singular cells on micro-posts ${ }^{23}$ and cell layers ${ }^{18,19}$ showed that they orient away from the flow at angles of up to $90^{\circ}$, i.e. perpendicular to the flow direction. To the best of our knowledge, an in depth study concerning the magnitude and orientation of the contraction under flow conditions has not yet been reported. However, Myers et $a .^{21}$ demonstrated that the orientation of force in platelets depends on the orientation they exhibit at the initial on-set of the flow with no change thereafter.

Here, we present a microfluidic flow chamber that allows us to mimic the shear rates found in blood vessels and simultaneously measure the forces generated by the platelets. Building on published fabrication methods (as discussed above), we combine a classical PDMS-glass microfluidic device with an elastic polyacrylamide (PAA) hydrogel coated with fibrinogen to facilitate platelet adhesion. We consider three different shear rates between $14 \mathrm{~s}^{-1}$ to $33 \mathrm{~s}^{-1}$ and compare the forces to those reported under no-flow conditions. We show that platelets do not alter the magnitude or temporal behavior of the generated forces when exposed to these low shear rates compared to noflow conditions and demonstrate that platelets do adapt to external shear stress in terms of force orientation. We explain this finding by simulating the stresses acting on the adherent cell under fluid flow.

\section{Materials and methods}

\section{Platelet isolation}

All experiments were performed in accordance with the Ethics Committee of the University Medical Center Göttingen, votum 11/11/09. The platelets were isolated from clinically expired human platelet preparations retrieved from healthy donors at the blood donation center of the University Medical Center Göttingen. The isolation procedure has been previously described elsewhere. ${ }^{5,16,27}$ Briefly, $4 \mathrm{~mL}$ of blood plasma was mixed with prostaglandin $\mathrm{E}_{1}$ (Cayman Chemical Company, Ann Harbor, MI, USA; final concentration $2.6 \mu \mathrm{g} \mathrm{mL}{ }^{-1}$ ). The solution was centrifuged for $20 \mathrm{~min}$ at $480 \times g$ and $21^{\circ} \mathrm{C}$ and the supernatant was removed. The cells were resuspended in $4 \mathrm{~mL}$ Pipes Saline Glucose (PSG: $5 \mathrm{mM}$ PIPES, $145 \mathrm{mM} \mathrm{NaCl}, 4 \mathrm{mM}$ $\mathrm{KCl}, 1 \mathrm{mM} \mathrm{MgCl} \cdot 6 \mathrm{H}_{2} \mathrm{O}, 5 \mathrm{mM}$ glucose, $0.05 \mathrm{mM} \mathrm{Na} \mathrm{HPO}_{4}$ ), followed by two additional centrifugation steps. After the last centrifugation step, the platelets were resuspended in HEPESTyrode buffer containing bovine serum albumin (BSA) (134 mM $\mathrm{NaCl}, 12 \mathrm{mM} \mathrm{NaHCO}, 2.9 \mathrm{mM} \mathrm{KCl}, 1 \mathrm{mM} \mathrm{MgCl}_{2}, 5 \mathrm{mM}$ HEPES, $5 \mathrm{mM}$ glucose, $0.34 \mathrm{mM} \mathrm{Na} \mathrm{HPO}_{4}$, supplemented with $5 \mathrm{mg} \mathrm{mL}{ }^{-1}$ BSA (Macs BSA stock solution, Miltenyi Biotech, Bergisch Gladbach, Germany)). The working solution was adjusted to $2 \times 10^{7}$ cells per mL. Thrombin (from human plasma, Sigma-Aldrich, St. Louis, Missouri, USA) solution was prepared in HEPES-Tyrode/BSA buffer at a final concentration of 40 units per $\mathrm{mL}$.

To visualize the actin structure in fully spread platelets, the cells were placed on fibrinogen coated cover slips and activated using thrombin at a final concentration of 4 units per mL. After 30 min of incubation, the cells were fixed using $4 \%$ paraformaldehyde. After thoroughly washing the samples in HEPES-Tyrode/BSA buffer, the platelets were incubated with phalloidin STAR 635 (Abberior GmbH, Göttingen, Germany; concentration of $0.11 \mu \mathrm{M}$ in phosphate buffered saline (PBS) containing 1\% goat serum) for $30 \mathrm{~min}$.

\section{Polyacrylamide substrate fabrication}

The polyacrylamide (PAA) substrates were produced similarly to previously reported protocols. ${ }^{6,16}$ A $24 \mathrm{~mm}$ by $60 \mathrm{~mm}$ glass cover slip (thickness No 1 (130 to $160 \mu \mathrm{m}$ ); VWR, Radnor, PA, USA) was treated with $0.1 \mathrm{M} \mathrm{NaOH}$, followed by a 5 min incubation with APTMS (3-aminopropyltrimethoxysilane; SigmaAldrich). Subsequently, the cover slip was rinsed with ultrapure water, dried, treated with $0.5 \%$ glutaraldehyde (Polyscience Inc., Warrington, PA, USA) in PBS and incubated for $30 \mathrm{~min}$. An additional glass cover slip (thickness No 1; VWR) was cut to a size of $3 \mathrm{~mm}$-by- $24 \mathrm{~mm}$, creating a glass strip, treated with Repel Silane (GE Healthcare, Little Chalfort, UK) for 5 min and rinsed first with $70 \%$ ethanol followed by ultrapure water. For the PAA solutions, we added $2.5 \mathrm{~mL}$ of $40 \%$ acrylamide (Bio-Rad Laboratories Inc., Hercules, CA, USA) and $1.3 \mathrm{~mL}$ of $2 \%$ bis-acrylamide (Bio-Rad) to $1 \times$ PBS (total volume $10 \mathrm{~mL}$ ). To produce $500 \mu \mathrm{L}$ volume of working solution, $15 \mu \mathrm{L}$ of $40 \mathrm{~nm}$ fluorescent beads, at a concentration of $0.2 \%$ solid (FluoSpheres, carboxylate-modified microspheres, 505/515 nm, Thermo Fisher Scientific Inc., Waltham, MA, USA), was added to the PAA solution. $2.16 \mu \mathrm{L}$ of the PAA solution including TEMED $\left(N, N, N^{\prime}, N^{\prime}\right.$-tetramethylethane-1,2-diamine; $0.5 \mu \mathrm{L}$; Bio-Rad) and APS (ammonium persulfate, Bio-Rad; final concentration of $4.4 \mathrm{mM}$ ) were added to the small glass stripe and the larger 
cover slip was positioned on top of it. The solution was left to polymerize for $60 \mathrm{~min}$ in this "upside-down" manner with the larger slide being supported by spacers such that the smaller glass slide did not touch the bench, thus avoiding pressure on it. The polymerized PAA gel was thus covalently bound to the rectangular glass slide. After removal of the small cover slip, we were left with a narrow stripe of PAA gel attached to the larger cover slip. The gel was incubated twice with Sulfo-SANPHA (0.4 $\mathrm{mM}$ in HEPES (2-[4-(2-hydroxyethyl)piperazin-1-yl]ethanesulfonic acid), Thermo Fisher Scientific Inc.) for $8 \mathrm{~min}$ each under UV light (wavelength $365 \mathrm{~nm}$ ), to achieve a uniform coverage. Finally, fibrinogen $\left(0.1 \mathrm{mg} \mathrm{mL}^{-1}\right.$ in ultrapure water, Calbiochem-Merck KGaA, Darmstadt, Germany) was added to the gel and incubated over night at $4{ }^{\circ} \mathrm{C}$. The substrates were kept at $4{ }^{\circ} \mathrm{C}$ submerged in PBS until further use. The finished substrates had a stiffness of $41 \mathrm{kPa}$ as determined by rheology and an average height of $30 \mu \mathrm{m}$. We chose a stiffness which: corresponds to a physiological environment, is stiff enough to not deform during our applied fluid flow, and where force measurements exist for the no flow case. ${ }^{6}$

\section{Microfluidic device design and fabrication}

Two separate devices were produced: a mixing channel and a measuring chamber. The mixing channel consisted of a three inlet, one outlet structure with a rectangular cross-section of $200 \mu \mathrm{m}$ width and $180 \mu \mathrm{m}$ height. The distance between the crossing point of the inlet channels and the outlet was $22.3 \mathrm{~mm}$. The device was fabricated by standard soft lithography methods. ${ }^{28,29}$ Briefly, SU-8 2150 photo resist (MicroChem, Newton, MA, USA) was spin-coated onto 2 inch silicon wafers (MicroChemicals, Ulm, Germany). After the soft bake step, the wafer was brought in contact with the photomask (Selba S.A., Versoix, Switzerland) and exposed to UV light (365 nm) using a mask-aligner (MJB4 Mask-Aligner, Süss MicroTec AG, Garching, Germany). A PDMS replica of the structured silicon wafer was produced by mixing PDMS and its cross-linker (Dow Corning, Midland, MI, USA) at a ratio of $10: 1$ and baking it for $1 \mathrm{~h}$ at $65^{\circ} \mathrm{C}$. In order to bond the PDMS replica to a glass cover slip (thickness No 1, VWR), we removed it from the wafer, punched a hole at each inlet as well as the outlet (biopsy puncher, $0.75 \mathrm{~mm}$ diameter, World Precision Instruments, Sarasota, FL, USA), treated both PDMS and glass with oxygen plasma for $20 \mathrm{~s}$ and pressed both surfaces together.

The measuring chamber was designed with a rectangular cross-section of height $110 \mu \mathrm{m}$ and width $4 \mathrm{~mm}$. The chamber length was set to $29 \mathrm{~mm}$. The fabrication process of the chamber is shown step-by-step in Fig. 1a. In this case, we used SU8-3050 negative photo resist for structuring the silicon wafers (step 1). Again, PDMS replicas were cast (step 2) and removed from the wafers (step 3). Holes were punched into the far ends of the channel structure in PDMS for the in- and outlet (biopsy puncher, $0.75 \mathrm{~mm}$ diameter). In order to obtain a tight seal, we used plasma treatment-aided bonding in this case as well. The cover slip containing the PAA gel, as described above, was first rinsed thoroughly with ultrapure water before all fluid was removed using a low lint tissue (KimTech Science low lint

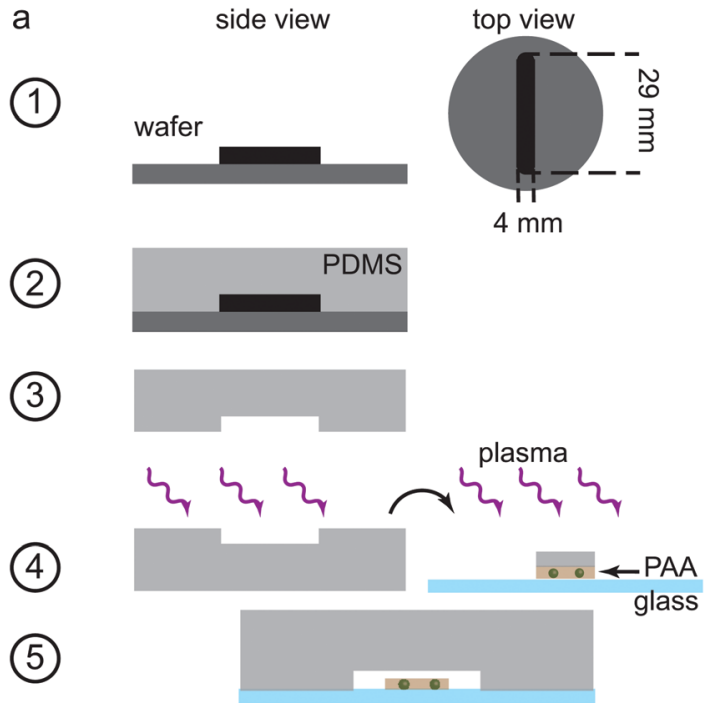

b

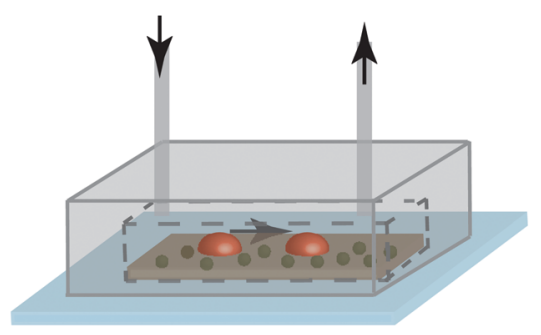

Fig. 1 Fabrication of the measuring chamber. (a) Step-by-step explanation of the fabrication of the measuring chamber. From a silicon wafer (dark gray with black resist structure, step 1), a PDMS replica (light gray, step 2-3) was produced. During oxygen plasma treatment (purple), the glass slide containing the PAA gel was protected by a stripe of PDMS (step 4). After plasma treatment, both parts were brought in contact to bond the final device (step 5). (b) Schematic of the final measuring chamber. The PAA substrate (brown) containing the fluorescent beads (green) is located on the glass slide; platelets (red) attach to the PAA gel. Sketches not to scale.

precision wipes, Kimberley-Clark Corp., Irving, TX, USA). A small PDMS stripe of similar dimensions as the PAA substrate, wetted with ultrapure water was placed onto the gel for protection from the oxygen plasma. Both the PDMS replica and the glass slide including the protected PAA gel were plasma oxidized for $10 \mathrm{~s}$ (step 4). The PDMS stripe was removed and the PDMS channel structure and the gel stripe were aligned by eye. The two components were pressed together to ensure a tight bonding (step 5). Polyethylene tubing (inner diameter $0.38 \mathrm{~mm}$, Intramedic Clay Adams Brand, Becton Dickinson and Company, Sparks, USA) was inserted at the inlet and outlet and the entire chamber, including the tubing, was flushed with ultrapure water, thus excluding all air bubbles. The tubing was sealed by melting it at the very end and the chambers were stored at $4{ }^{\circ} \mathrm{C}$ until further use. The final chamber, as used in the experiments, is shown schematically in Fig. $1 \mathrm{~b}$.

\section{Experimental setup and imaging}

The mixing device was connected to the measuring chamber via tubing such that the outlet of the mixing device was connected 
to the inlet of the measuring chamber. The average length of the connecting tube was $12 \mathrm{~cm}$. The outlet tubing of the measuring chamber was opened and through this entrance, the entire system was flushed with HEPES-Tyrode/BSA buffer. For imaging the fluorescent markers and platelets, an IX 81 inverted microscope (Olympus, Hamburg, Germany) equipped with a $60 \times$ oil-immersion objective (UPlanSApo, NA 1.35, Olympus), a QImaging Retiga 6000 CCD camera (QImaging, Surrey, BC, Canada) and an incubation chamber (INUG2EONICS, Tokai Hit Ltd. Co., Shizuoka, Japan) was used. The measuring chamber was fastened inside the incubation chamber and the mixing device just outside on the microscopy stage. The tubings at the inlets were connected to Hamilton gas-tight syringes (series 1000, Hamilton, Bonaduz, Switzerland; total volume of $250 \mu \mathrm{L}$ for the thrombin-filled syringes and $2 \mathrm{~mL}$ volume for the platelet-filled syringe) such that thrombin was injected through both side inlets and the platelet solution through the central inlet. The thrombin was employed to activate the platelets and they were incubated with the reagent for two to five minutes in total, comparable to previous experiments without flow. ${ }^{6}$ The flow rates were set such that the volume ratio between the (combined) thrombin syringes and the platelet solution syringe was 1:9. This resulted in a dilution of the inlet thrombin concentration from 40 units per $\mathrm{mL}$ to a final outlet concentration of 4 units per $\mathrm{mL}$, which is well within the physiological range, ${ }^{30}$ thus ensuring complete activation of the platelets. ${ }^{6,27}$ This solution was fed into the measuring chamber. The syringes were driven by a syringe pump system (neMESYS, Centoni $\mathrm{GmbH}$, Korbuß en, Germany). The microscopy recording was started when all liquids, by estimation of the traveling time, had reached the mixing region in the mixing device. As estimated from the remaining channel length and length of the connecting tubing as well as the flow velocity, from this time point on, the cells reached the measuring chamber within 5 min. Recordings were performed using a FITC/Cy5 dual-band filter (excitation maxima at $470 \mathrm{~nm}$ and $628 \mathrm{~nm}$, AHF Analysentechnik AG, Tübingen, Germany) and an emission filter of wavelength $537 \mathrm{~nm}$ and $694 \mathrm{~nm}$ (AHF Analysentechnik AG). As light source for the fluorescent recordings we used a xenon arc lamp (MT-ARC/XE, Olympus), set to $23 \%$ intensity and an exposure time of $50 \mathrm{~ms}$. The platelets were tracked employing bright-field illumination, set to $3.2 \mathrm{~V}$ and an exposure time of $500 \mathrm{~ms}$. An entire recording amounted to $1.5 \mathrm{~h}$ with a frame rate of $0.13 \mathrm{fps}$ (time interval between frames $7.5 \mathrm{~s}$ ). For actin stained, fixed platelet samples, an oil-immersion $100 \times$ objective (NA 1.4, Olympus) was used with an illumination time of $100 \mathrm{~ms}$ for fluorescence and phase contrast images. As mentioned above, the recording was started before the platelets reached the measuring chamber. Thus, the time point of attachment could be determined from the movies as the time point at which the discoid platelets come into focus and stopped moving with the flow. A rolling of the platelet on the surface was not observed. The recording was stopped after a total recording time of $1.5 \mathrm{~h}$ was reached. Due to the different attachment time points of the platelets, the entire observation time varied between individual cells but was always at least $10 \mathrm{~min}$.

\section{Calculation of force}

The recorded bead patterns were analyzed using a specially adapted TFM method that accounts for the highly dynamic force evolution of platelets. ${ }^{6}$ For comparison between the different measurements, we calculated the total force $F_{\text {tot }}(t)$ at a given time point within a region of interest (ROI):

$$
F_{\text {tot }}(t)=\int_{\text {ROI }}|\mathbf{T}(\mathbf{x}, t)| \mathrm{d} A,
$$

with the traction force $\mathbf{T} .{ }^{31-34}$

To determine the degree of anisotropy in the contraction of the platelets, as well as the contraction orientation compared to the flow, we calculated the force dipole of each cell. The force dipole tensor $\mathbf{P}$ is defined via the force density ${ }^{35-37}$ which corresponds to the traction forces and is thus given by

$$
\mathbf{P}=\int \mathbf{x} \cdot \mathbf{T}(\mathbf{x}) \mathrm{d} \mathbf{x} .
$$

The eigenvectors of $\mathbf{P}$ then describe the orientation of the dipole axes of the platelet and the corresponding eigenvalues $D_{1}$ and $D_{2}$, with $D_{1} \geq D_{2}$, provide the corresponding dipole magnitudes. We define the degree of anisotropy as the ratio between the eigenvalues

$$
q=\frac{D_{1}}{D_{2}}
$$

Note that $q \in[1, \infty)$ where $q=1$ corresponds to an isotropically contracting platelet. The degree of anisotropy averaged over time for each cell was determined using the temporal variance of $q$ as described previously. ${ }^{6}$

By definition, the major dipole axis (the eigenvector corresponding to $D_{1}$ ) describes the axis between the highest, opposing traction force "hot spots". Hence, this axis was interpreted as the preferred contractile orientation of the platelet. The flow direction was tracked using the bright-field images recorded of platelets that were passing by, and specifically a region where no cell were attached to prevent any influence on the flow field (see ESI, $\dagger$ Fig. S1a). The angle between the preferred contractile orientation and the flow direction was then defined as the angle between the major dipole axis and the flow direction. Note that only angles between $0^{\circ}$ and $90^{\circ}$ needed to be considered due to the constant flow profile parallel to the substrate (see ESI, $\dagger$ Fig. S2 for more information). Due to the stability of the flow direction and the preferred contractile orientation, the angles between the mean flow direction and the major dipole axis were averaged over all time points (ESI, $\dagger$ Fig. S1b).

\section{Simulations}

All simulations were performed using the finite element methods (FEM) software package COMSOL Multiphysics 5.3 (COMSOL Multiphysics AB, Stockholm, Sweden).

\section{Flow simulations}

To reduce the calculation time, both the mixing channel and measurement chamber geometry were cut along the vertical symmetry axis. The flow within the channels was calculated by 
assuming a laminar flow profile, resulting in the stationary Navier-Stokes equation

$$
\rho(\mathbf{v} \cdot \nabla) \mathbf{v}=-\nabla p+\eta \Delta \mathbf{v}
$$

with the velocity $\mathbf{v}$, the density $\rho$, the pressure $p$ and the dynamic viscosity $\eta .^{38}$

For the mixing channel, the concentration distribution of platelets and thrombin was determined using the convectiondiffusion equation of mass transport

$$
\frac{\partial c}{\partial t}=\nabla \cdot(D \nabla c)-\nabla \cdot(\mathbf{v} c)
$$

with the concentration of the species $c$ and the diffusion coefficient $D$. The diffusion coefficient for the unspread platelets was estimated from their size (about $3 \mu \mathrm{m}$ in diameter for a spherical shape) by the Stokes-Einstein relation

$$
D=\frac{k_{\mathrm{B}} T}{6 \pi \eta r},
$$

where $k_{\mathrm{B}}$ is the Boltzmann constant, $T$ the temperature and $r$ the radius of the platelet, to about $1.63 \times 10^{-13} \mathrm{~m}^{2} \mathrm{~s}^{-1}$. For thrombin, we used a value for the diffusion coefficient from literature, $8.7 \times 10^{-11} \mathrm{~m}^{2} \mathrm{~s}^{-1} \cdot{ }^{39}$ The differential equations were solved within the volume of the mixing channel using the segregated solver implemented in Comsol Multiphysics, where the subsystems are solved by GMRES (generalized minimal residual method). In total, the system included about 960000 degrees of freedom.

For the measuring chamber, we additionally determined the shear rate on top of the substrate surface. The shear rate tensor $\dot{\Gamma}$ is given by $^{38,40}$

$$
\dot{\Gamma}=\nabla \mathbf{v}+(\nabla \mathbf{v})^{T},
$$

with it's norm given as

$$
\dot{\gamma}=|\dot{\Gamma}|=\sqrt{\frac{1}{2} \dot{\Gamma}: \dot{\Gamma}},
$$

where the "contraction operator" is defined by $\mathbf{a}: \mathbf{b}=\Sigma_{n} \Sigma_{m} a_{n m} b_{n m}$. The shear rate and the shear stress $\tau$ are related to each other by ${ }^{38}$

$$
\tau=\eta \dot{\gamma}
$$

The discrete system of the measuring chamber was solved by using a stationary, algebraic multigrid solver with a total of about 6.6 million degrees of freedom.

\section{Deformation simulations}

To describe the strain caused by the stresses acting on the platelet during attachment, we simulated the interaction between an unspread, attached platelet and the laminar flow. Here, the platelet was represented by an elastic spherical cap (height $1 \mu \mathrm{m}$, radius of curvature $1.5 \mu \mathrm{m}$, elastic modulus $5 \mathrm{kPa}$, Poisson's ratio of 0.3 , and density $1.1 \times 10^{3} \mathrm{~kg} \mathrm{~m} \mathrm{~m}^{-3}$ ). We approximated the substrate $(41 \mathrm{kPa})$ as a stiff solid, which is a valid assumption as in the experiment the stiffness was chosen such that the substrate did not deform by the flow.
To reduce computing time, only a region of interest (chamber length $200 \mu \mathrm{m}$ ) within the measuring chamber was simulated, long enough to establish a laminar flow profile before reaching the platelet. Apart from calculating the shear stresses within the flow, we also determined a scalar measure for the entire stress tensor defined on the cell volume, the so called von Mises stress $\sigma_{\text {Mises }}$, or effective stress, defined as ${ }^{41}$

$$
\sigma_{\text {Mises }}=\sqrt{\frac{3}{2} \sum_{i=1}^{3} \sum_{j=1}^{3} \sigma_{i j}{ }^{2}-\frac{1}{2} t^{2}}
$$

with the three dimensions $i, j, k$, being the corresponding components of the stress tensor and $t=\operatorname{trace}(\sigma)=\sum_{k=1}^{3} \sigma_{k k}$. The solution of the differential equations was implemented in Comsol Multiphysics using the segregated solver where the subsystems were solved by GMRES. In total, the system included about 1.1 million degrees of freedom.

\section{Results and discussion}

\section{Characterization of the microfluidic system}

Before entering into the measuring chamber, the platelet solution was mixed with thrombin in a controlled way within the mixing channel. To understand this mixing procedure, simulations were conducted as described above. Simulation results for the concentration distributions within the mixing region as well as the first $1.5 \mathrm{~cm}$ of the outlet channel are shown in Fig. 2a for a flow rate of $700 \mu \mathrm{L} \mathrm{h} \mathrm{h}^{-1}$. Due to the pronounced difference in size and therefore in diffusion coefficient between platelets $\left(1.63 \times 10^{-13} \mathrm{~m}^{2} \mathrm{~s}^{-1}\right)$ and thrombin $\left(8.7 \times 10^{-11} \mathrm{~m}^{2} \mathrm{~s}^{-1}\right),{ }^{39}$ the cells diffuse considerably more slowly towards the channel walls than does the thrombin towards the center of the channel. We thus defined the longitudinal channel position of complete mixture $l_{\text {mix }}=10 \mathrm{~mm}$ as the position at which the concentration of the thrombin was equally distributed within the channel cross-section, i.e. all platelets experience the same thrombin concentration ( $30 \mathrm{mM}$, corresponding to 4 units per $\mathrm{mL}$ ). $l_{\mathrm{mix}}$ is equivalent to the position at which the thrombin concentration at the central line of the channel reaches a constant level (Fig. 2b). The incubation time of platelets in thrombin was hence defined as the time interval needed for the fluid to travel between $l_{\text {mix }}$ and the entrance into the measuring chamber. Considering the remaining channel length and the length of the connecting tube to the measuring chamber, this time interval amounted to about $3 \mathrm{~min}$. This was comparable to the reaction time used for no-flow experiments reported previously. ${ }^{6}$ Activation of platelets (in our case by thrombin) is a prerequisite for spreading, which in turn was clearly observed in our experiments. Although the highest shear rate within the channel (300 $\mathrm{s}^{-1}$ for the fastest flow rate, shown in Fig. 2c) was higher than in the measuring chamber as described below (maximum of $33 \mathrm{~s}^{-1}$ ), it was well within physiological values and indeed one order of magnitude lower than comparable 
a
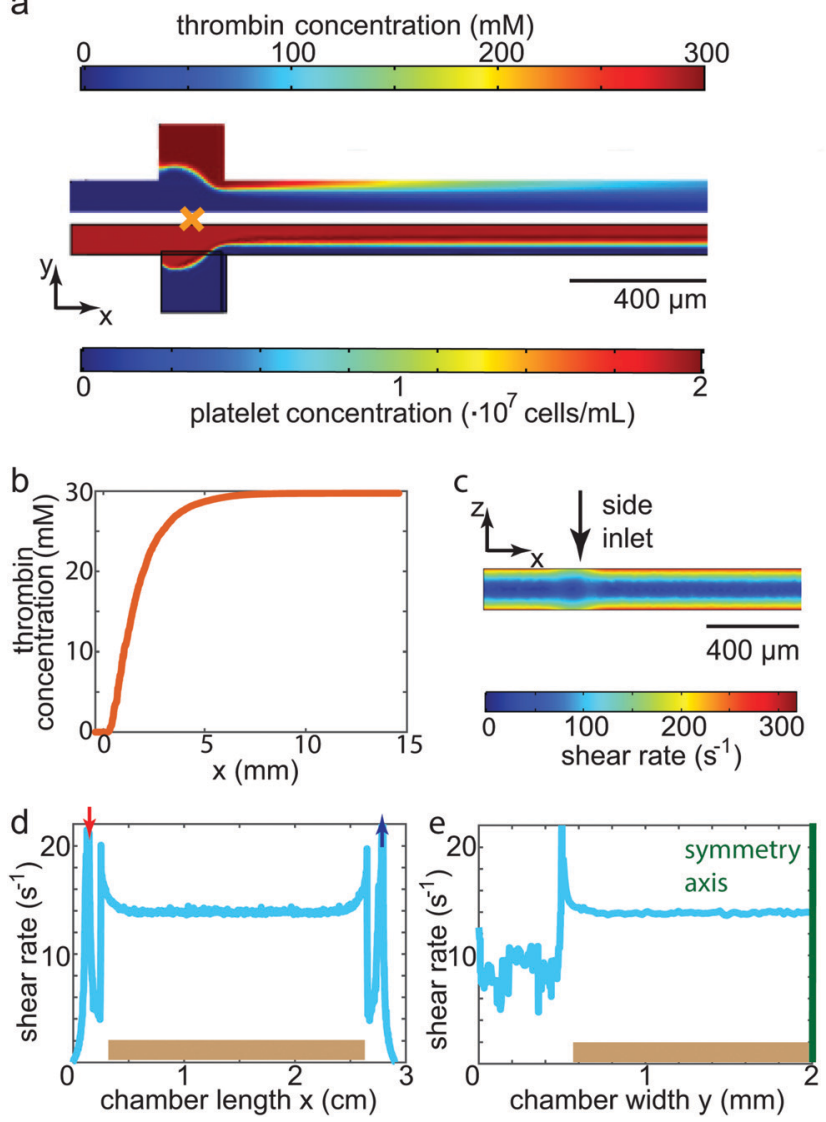

Fig. 2 Characterization of the microfluidic devices. (a) FEM simulation of the concentration profiles in the mixing channel (first $1.85 \mathrm{~mm}$ are shown); top: concentration of thrombin; bottom: concentration of platelets. (b) Increase of the thrombin concentration in flow direction for the first $15 \mathrm{~mm}$ of the mixing channel, taken along the center line of the channel with the origin located at the orange cross in panel a (center of the side inlet). The concentration stabilizes within the first $10 \mathrm{~mm}$, thus defining the point of complete mixture $I_{\text {mix }}$. (c) FEM simulation of the shear rate in the central plane of the mixing channel. All simulations in the mixing channel $(a-c)$ shown here were conducted at a total flow rate of $700 \mu \mathrm{L} \mathrm{h}^{-1}$. (d and e) Shear rate in the measuring chamber directly above the substrate (brown), i.e. where the platelets adhere (flow rate $300 \mu \mathrm{L} \mathrm{h}^{-1}$ ). Inlet: red arrow, outlet: blue arrow. (d) Shear rate in flow direction; (e) shear rate perpendicular to the flow direction. The symmetry axis is marked in green. Note that the channel length is given in units of $\mathrm{cm}$ and the width in units of $\mathrm{mm}$. All shear rates scale linearly with the respective flow rate. Noise in the simulated shear rate is due to numerical errors in the simulation.

values found in arteries, thus avoiding additional physical activation of the platelets by shear.

A measuring chamber suitable to realistically perform force measurements on platelets under flow needs to fulfill several criteria. First, a controlled fluid flow environment needs to be combined with an elastic substrate for TFM. Second, the chamber has to be reliable, reproducible and leak-tight. Third, the resulting chamber should create a physical environment comparable to that of a human blood vessel. To characterize the chamber and test its comparability with physiological conditions, we simulated the fluid flow in the chamber as described above. For physiological conditions, we determined the shear
Table 1 Physical characteristics of the flow within the measuring chamber. For the different flow rates, the mean velocity in the chamber as well as the shear rate and shear stress directly above the substrate are listed

\begin{tabular}{llll}
\hline $\begin{array}{l}\text { Flow rate } \\
\left(\mu \mathrm{L} \mathrm{h}^{-1}\right)\end{array}$ & $\begin{array}{l}\text { Mean velocity } \\
\left(\mu \mathrm{m} \mathrm{s}^{-1}\right)\end{array}$ & $\begin{array}{l}\text { Shear } \\
\text { rate }\left(\mathrm{s}^{-1}\right)\end{array}$ & $\begin{array}{l}\text { Shear } \\
\text { stress }(\mathrm{Pa})\end{array}$ \\
\hline 300 & 236 & 14 & 0.014 \\
500 & 393 & 23 & 0.023 \\
700 & 550 & 33 & 0.033
\end{tabular}

rates such that they corresponded to those found in large human veins. Biologically, at these low shear rates, it has been shown that the main mechanism leading to platelet attachment and aggregation is the interaction of fibrinogen and the integrin $\alpha$ IIb $\beta 3 .{ }^{11}$ Hence, the substrates were coated with fibrinogen for this study. As the platelets are, in their spread state, only about $100 \mathrm{~nm}^{4,42}$ high and thus considerably smaller than the channel structure itself, we assumed that the cells were effectively exposed to a shear rate as found on the surface of the substrate. The shear rate at the top of the elastic gel is constant, both along the length as well as the width of the chamber, as shown in Fig. $2 \mathrm{~d}$ and e, respectively. In this particular example, we show the results for a flow rate of $300 \mu \mathrm{L} \mathrm{h}^{-1}$, resulting in a shear rate of $14 \mathrm{~s}^{-1}$. The shear rates for all three flow rates used in our study are listed in Table 1 alongside the corresponding mean velocities and shear stresses at the substrate surface found in the chamber. The constant shear rate profile on top of the PAA gel is a great advantage for our measurements as all platelets attaching and spreading on the gel experience equivalent force fields. Such a situation cannot be realized using the micro-post arrays as those show a varying profile between and on the micro-post themselves. ${ }^{23}$ Comparing the flow conditions to physiological conditions, all shear rates used here were on the order of $10 \mathrm{~s}^{-1}$ and thus similar to the shear rates found in large veins in the human body. To verify the values for the shear rates determined by simulation, we also conducted experiments to determine the shear rate by studying the flow within a chamber when no cells were present. We could show that the simulated and real values for the shear rates differed by less than $5 \%$ (compare ESI, $\uparrow$ Section S3). Our chamber proved to be reliable in terms of leakage. By plasma treating both the glass slide containing the substrate and the PDMS cast, a tight, covalent bond was formed. The application of a moist PDMS stripe on top of the substrate during plasma treatment avoided the drying out of the hydrogel. At the same time, it protected the fibrinogen during plasma treatment, ensuring that it was not damaged and platelets readily attached and spread on the substrate.

\section{Traction force distribution and total force development}

For each of the different flow rates, on average, data of 22 platelets from three independent experimental days were analyzed concerning spatial traction force distribution as well as the temporal development of the total force. As a baseline, we considered the data recorded for platelets without flow reported previously. ${ }^{6}$ Fig. 3a shows the averaged total force for the first $30 \mathrm{~min}$ of recording for each flow rate. We observe 
a

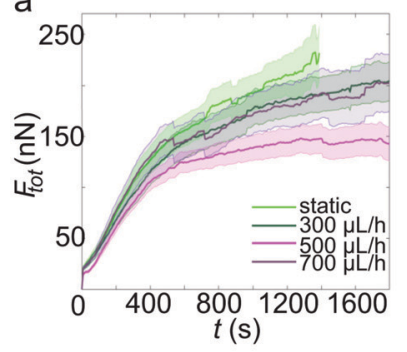

C

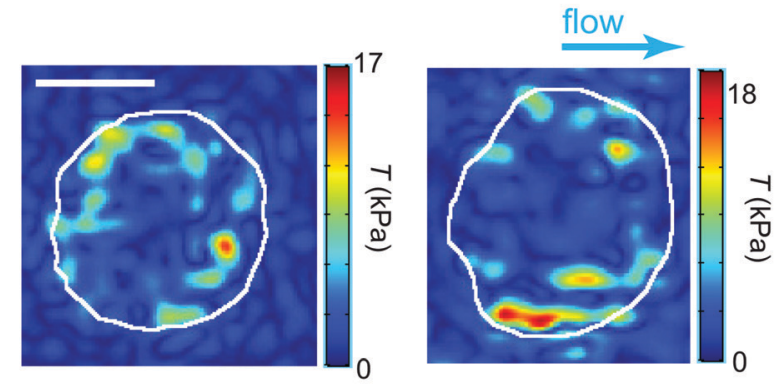

d

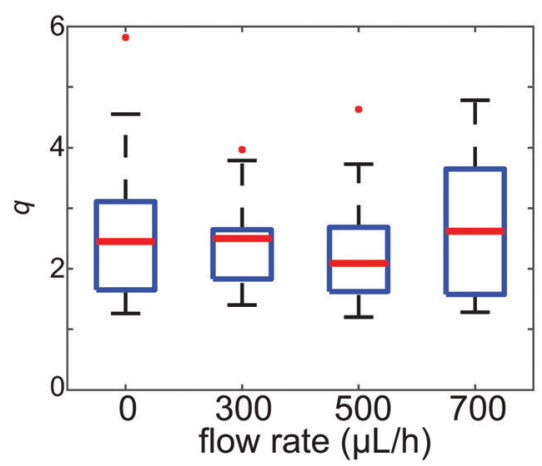

Fig. 3 TFM results for platelets under shear flow. (a) Average total forces for the first $30 \mathrm{~min}$ of recording. The bold lines denote the average curves, the transparent areas show the corresponding standard errors. (b) Maximum force of each individual platelet plotted against the final spread area. The black line represents the fit to the model. ${ }^{43}$ (c) Examples of traction force patterns recorded under flow conditions. The white lines denote the periphery of the final spread cell. We observe force hot spots near the circumference of each platelet. The flow direction is shown by the blue arrow. Scale bar: $5 \mu \mathrm{m}$. (d) Dipole ratio (degree of anisotropy) $q$ distribution for each flow rate. No significant difference is observed for the different data sets.

that the magnitude of the total force is independent of the shear rate. However, it should be noted that the temporal behavior varied greatly between the single platelets within the same group, both in magnitude as seen in the standard errors included in Fig. 3a, and the overall behaviour (see ESI, $\dagger$ Fig. S5). The maximum magnitude of the total force was independent of the flow rate, but increased with increasing platelet size as shown in Fig. 3b. The black line denotes the fit of all data to a model that considers a contracting, elastic, disc-like cell as derived by Edwards and Schwarz. ${ }^{43}$ Here, the spread cell is given by a radius $r_{0}$, a height $h_{\mathrm{c}}$, an elastic modulus $E_{\mathrm{c}}$, a Poisson's ratio $\nu_{\mathrm{c}}$ and an internal stress $\sigma_{0}$. The decay of the traction forces in space is then defined by the localization length $l_{\mathrm{L}}$ which depends on $E_{\mathrm{c}}, \nu_{\mathrm{c}}$ and $h_{\mathrm{c}}$ as well as the elastic properties of the substrate and the adhesion layer. $\sigma_{0}$ and $l_{\mathrm{L}}$ are used as free fit parameters. ${ }^{6}$ Using the data depicted in Fig. $3 \mathrm{~b}$ for the values $r_{0}$ and $F_{\text {tot }}$ as well as $\nu_{\mathrm{c}}=0.3$ and $h_{\mathrm{c}}=100 \mathrm{~nm}$, we obtain $\sigma_{0} \approx 150 \mathrm{kPa}$ and $l_{\mathrm{L}} \approx 1.3 \mu \mathrm{m}$, very similar to the values for the no-flow conditions. We thus conclude that the low shear rates studied here do not influence the platelet in its spread state in terms of the level of force generation or internal stress.

Fig. 3c shows two typical examples of spatial traction distributions with the distinct force hot spots typical for platelets. ${ }^{17}$ Calculating the dipole ratio according to eqn (3) demonstrates that the contraction is slightly anisotropic (median 2.2, Fig. 3d). As determined for the no flow conditions previously, this dipole ratio cannot be explained by a shape anisotropy, since the platelets are almost circular. ${ }^{6}$ None of the considered four cases of flow rates was statistically significantly different from the others using the Wilcoxon rank sum test.

Taken together, these results show that neither the temporal evolution of the total force nor its maximum value, the spatial distribution or the anisotropy of the force fields are affected by shear rates up to $33 \mathrm{~s}^{-1}$. This is an interesting result as it indicates that platelets within this physiological environment in terms of shear flow either do not sense these comparatively low forces, or they are able to compensate internally for the external mechanical impact.

\section{Adaptation of contraction orientation to the flow direction}

The slight anisotropy observed in the force fields leads us directly to the question of how the preferred direction is related to the direction of the flow. As the preferred orientation of contraction, we defined the angle between the flow direction and the major dipole axis, i.e. the eigenvector corresponding to the larger eigenvalue of the dipole matrix $\mathbf{P}$ determined according to eqn (2). This is well justified as the major dipole axis due to its mathematical definition is parallel to the connecting line between the most prominent force hot spots. Both the direction of the flow as well as the dipole are stable over time within the error bounds due to the measurement noise (see ESI, $\dagger$ Fig. S1b and S3). Hence, we first determined the mean of all measurements of the flow direction before calculating the angle towards the dipole orientation. The resulting values for the angle over time were then averaged again so each platelet was assigned a single angle for the preferred contraction orientation. We show them in the rose histograms in Fig. $4 \mathrm{a}-\mathrm{c}$ for flow rates of $300 \mu \mathrm{L} \mathrm{h}^{-1}$ (a), $500 \mu \mathrm{L} \mathrm{h}^{-1}$ (b) and $700 \mu \mathrm{L} \mathrm{h}^{-1}$ (c), respectively. For the two lower flow rates, on average, the contraction orientation was found to be about $45^{\circ}$. Interestingly, by contrast, for the highest flow rate of $700 \mu \mathrm{L} \mathrm{h}^{-1}$, the angle increased to nearly $90^{\circ}$. The observed increase in angle between the highest and lower flow rates is significant as determined using the Wilcoxon rank sum test and independent of the corresponding dipole ratio. It is not possible to determine a preferred direction of orientation for the no flow conditions, as the reference direction, which is the flow direction, is missing. When determining the angle at which the platelets contract at no flow conditions with respect to the horizontal image axis, no preferred orientation is observed. Thus, a bias in our experimental setup or analysis code can be excluded. 
a

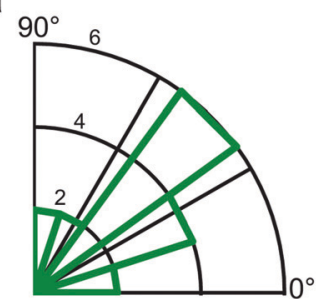

C

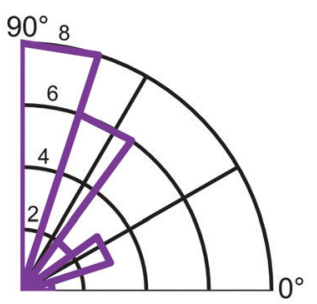

b

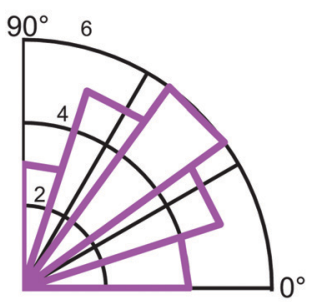

d

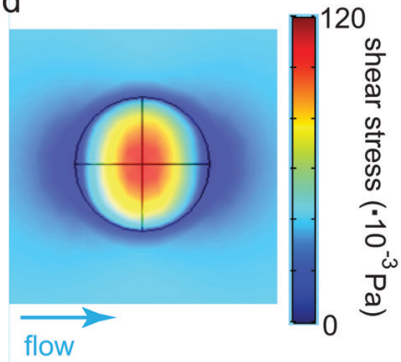

Fig. 4 Angle between the preferred orientation of contraction and the flow direction for $300 \mu \mathrm{L} \mathrm{h}^{-1}$ (a), $500 \mu \mathrm{L} \mathrm{h}^{-1}$ (b) and $700 \mu \mathrm{L} \mathrm{h}^{-1}$ (c). For the lower two flow rates, the average angle is about $45^{\circ}$, for the highest flow rate it is nearly $90^{\circ}$. (d) The shear stress acting on the elastic cell at the time point of attachment for a flow rate of $700 \mu \mathrm{L} \mathrm{h} \mathrm{h}^{-1}$. The flow direction is shown by the blue arrow.

\section{Simulation of stresses acting on adherent platelets}

To qualitatively understand the observed contraction behavior, we simulated an adherent, elastic platelet on the substrate as described above. As we measure a stable contraction angle in time, we conclude that the time point at which the final orientation is established can be approximated by the time point of attachment. Note that at this time point, no internal contraction forces are observed and the external forces on the cell determine its behavior. This was previously suggested by Myers et $a l^{21}$ when studying platelets under flow, although in this study, the flow was applied only after the attachment of the cells. We thus approximated an unspread cell with the same elastic parameters as for the model used in the fit shown in Fig. 3 b. We assume an elastic membrane fully adhered to the substrate, ignoring any specific internal structures such as e.g. actin fibers or integrin clusters. Note that because we consider the cell as a 3D object disturbing the flow, the shear rate, as defined by eqn (7), increases quite considerably in proximity of the cell's surface. The corresponding shear stresses of the fluid are shown in Fig. 4d and the regions of lowest stress (dark blue) are found in flow direction. Hence, the fluid's shear stress does not explain the perpendicular orientation of the platelets.

Instead, we consider the force that results from the fluid interacting with the cell. This force gives rise to a stress distribution at the cell surface and in turn deforms the cell. Note that the resulting stress defined on the cell volume is a three-by-three tensor. In order to consider all components of the stress tensor, we applied the concept of the von Mises stress as defined in eqn (10), borrowed from structural mechanics. $\sigma_{\text {Mises }}$ takes all stress components into account and summarizes them in a scalar entity, which can be plotted in a $2 \mathrm{D}$ map as
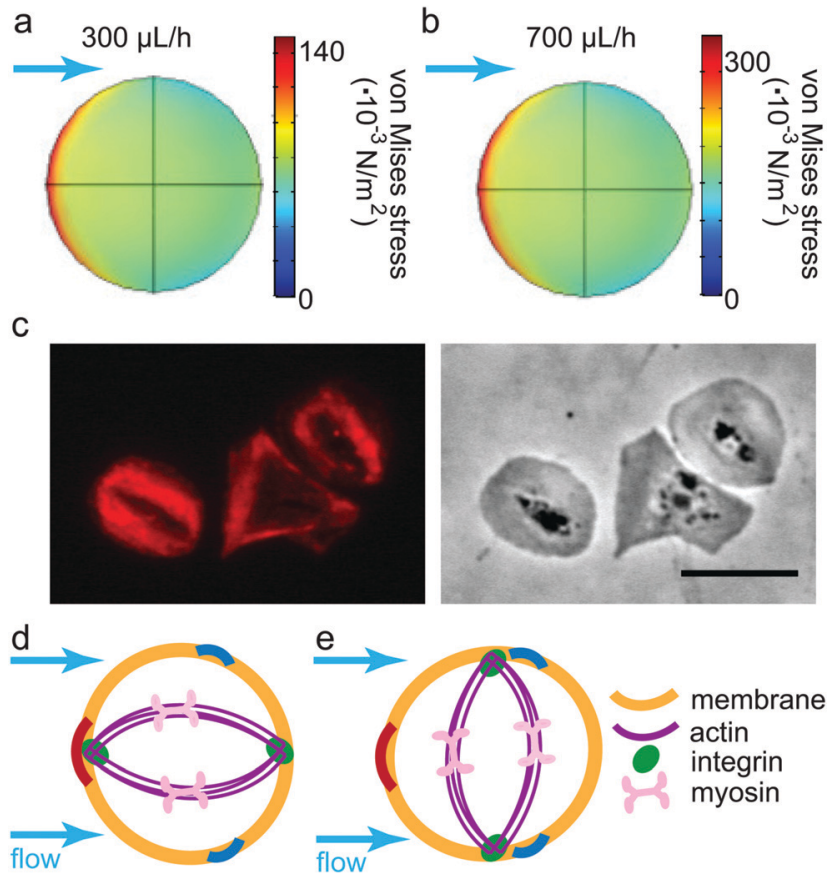

Fig. 5 The calculated von Mises stress distribution for the lowest (a) and highest (b) flow rates. The highest stresses are found pointing towards the flow while the lowest stresses are positioned at an angle of $>90^{\circ}$. (c) An example for typical actin structures (left, phalloidin staining, fluorescence microscopy) and membranes (right, bright field recording) of spread platelets. Note the two to three thicker actin bundles spanning through the entire cell. For a parallel orientation of the force-transmitting network, as shown in (d), one of the focal adhesions (green) is located at the point of highest von Mises stress (dark red arc). In a perpendicular orientation (e), both integrin clusters are found close to the lowest stresses (dark blue arcs). We thus argue that the perpendicular orientation is more stable in a flow environment than the parallel orientation. Note that the forcetransmitting network is reduced to four components in this sketch: the platelet membrane (orange), the thick actin bundles (purple) with bound myosin motors (pink) and the integrin clusters (green) which serve as anchor points. The flow is coming from the left hand side (blue arrows). Scale bar: $5 \mu \mathrm{m}$.

shown in Fig. $5 \mathrm{a}$ and $\mathrm{b}$ for the lowest and highest flow rate, respectively. Note that the distribution of the von Mises stresses differs considerably from the shear stress distribution within the fluid as shown in Fig. $4 \mathrm{~d}$ as the stresses at the platelet also depend on the cell's geometry. Irrespective of the flow rate, we find the regions of highest von Mises stress pointing towards the flow direction (color coded in red in the simulation) and the lowest stresses slightly behind the $90^{\circ}$ axis (color coded in blue). In structural mechanics, the von Mises stresses are used to determine the region where a structure would first yield under an applied load, known as the von Mises yield criterion. We here use this interpretation of the von Mises stress to combine the results from the simulations and the experimental observations. Specifically, we interpret the concept of a material "yielding" as the detachment of a contractile platelet from the substrate.

To be able to correlate the simulated von Mises stress distribution with the experimental data, we consider a very simplified model of the internal structure of an adherent platelet, 
taking into account the most prominent components for forcetransmission. For different scenarios of platelet orientation within the flow, they are sketched in Fig. $5 \mathrm{~d}$ and e. The platelet is bounded by a membrane (orange) and attached to the substrate at, for simplicity, two opposing focal adhesions (green). The thick actin bundles (purple), including myosin motors (pink), are shown to connect the focal adhesions. This internal organization has previously been reported for studies employing actin stainings ${ }^{44,45}$ (compare also Fig. 5c) or integrin force sensors. ${ }^{17}$ It was shown that actin fibers within platelets develop in two phases; first, the actin polymerizes into a scaffold-like actin structure, which is then reinforced and stress-fiber-like bundles appear. ${ }^{45}$ Similar time scales were observed for the force development of platelets using force sensors. ${ }^{17}$ Here, a lower force regime was observed during actin-polymerization driven spreading, whereas a higher force was observed after initial spreading, coinciding with the reported reinforcement of actin bundles. We argued previously that for the case of no flow the measurements on elastic substrates using traction force microscopy allow to observe the second phase but not necessarily the first. ${ }^{6}$ Hence, to understand the structure of a platelet under flow in connection to the measured forces, we can safely assume the case of a fully spread cell with defined actin structures as shown in Fig. 5.

We can now consider two limiting cases in terms of network orientation with respect to the flow direction. (i) If the orientation of contraction is parallel to the flow (Fig. 5d), one of the focal adhesions is located at the point of highest stress (dark red arc). With increasing flow rate, this location corresponds to the point of most probable breakage of the connection to the substrate. (ii) If the cell, however, adapts a perpendicular orientation (Fig. 5e), both focal adhesions are close to the points of lowest stress (dark blue arcs). We thus conclude that this configuration is most stable with respect to breakage of the focal adhesions under shear flow.

This simple model explains our observations shown in Fig. 4. At comparatively low shear rates, i.e. up to $23 \mathrm{~s}^{-1}$, the cell starts to orient away from the flow direction, hence the angle of approximately $45^{\circ}$. At higher shear rates, however, the angle of contraction increases to about $90^{\circ}$, corresponding to the most stable situation according to the calculated von Mises stresses. We thus assume that beyond some threshold shear rate, which lies between $23 \mathrm{~s}^{-1}$ and $33 \mathrm{~s}^{-1}$, the cytoskeletal structures, consisting of actin, myosin and focal adhesions, are built up directly after platelet attachment in such a way that the mechanically most stable situation is ensured. This strongly indicates that already at low physiological shear rates, platelets align in such a way that the resulting blood clot is as stable as possible in terms of attachment to reduce the possibility of wound breakage. To validate our hypothesis about the correlation of the actin orientation under flow, further studies will have to be conducted that simultaneously visualize the actin dynamics in living platelet. A related observation was reported recently by a combination of fluorescence polarization techniques with molecular force microscopy. ${ }^{46}$ Here, the authors observed that the forces generated by platelets orient at an angle of $90^{\circ}$ to the polarized, incident light.
A similar contraction behavior under flow conditions was reported for endothelial cells when studying the process of angiogenesis, ${ }^{25}$ i.e. the formation of new blood vessels. Similar to the attachment of blood platelets, this phenomenon occurs under blood flow conditions. Hence, experiments were conducted using layers of already attached endothelial cells. ${ }^{18,19,23}$ For different shear stresses ranging from 0.014 $\mathrm{Pa}$ to $2 \mathrm{~Pa}$ and different chamber geometries, the preferred direction of contraction with respect to the flow was determined. For comparison, the attached, unspread platelets in our geometry experience a maximum shear stress between 0.05 $\mathrm{Pa}$ and 0.12 $\mathrm{Pa}$ on top of the cell (for the shear stresses within the empty channel, refer to Table 1), depending on the flow velocity. Note that the shear stress on top of the unspread platelet is higher than the shear stress found within the empty channel due to the disturbance in the flow and the resulting increase of the velocity gradient at the cell boundary layer. Whereas all studies performed on endothelial cells reported a large variance in the angle, a general tendency towards $90^{\circ}$ was observed, in agreement with our results. The variance in angle suggests that this response is not an "all-or-nothing" process but the cells adapt gradually to the shear stress, just like our results show for different flow rates.

The forces we exert on the cells by flow are low compared to the forces generated by platelets once they spread and contract (150 nN, see Fig. 3). Thus, one important question is why the platelets still react to these low forces. While we do not have a final answer to this question, one may speculate that at the moment of attachment, when the preferred direction of contraction is determined, the internal contraction forces are still low and thus do not influence the cell behavior. Additionally, the focal adhesions are not yet fully developed and as stable as they are later and thus more sensitive to low forces.

The results are also reminiscent of studies where the gradual adaptation to external stress was demonstrated by repeated stretching of cells. ${ }^{47}$ Endothelial cells were cultured on elastic substrates which were then stretched in a cyclic manner. It was shown that the actin stress fibers oriented in a perpendicular direction to the stretch direction. The orientation was dependent on the magnitude of the substrate deformation and a threshold had to be exceeded to align all actin fibers towards this direction. Below this threshold, larger variations in orientation were seen, again indicating that the alignment is a gradual process. This finding agrees well with our results here.

However, a perpendicular orientation of cells stretched on elastic substrates is not always observed. For fibroblasts, it could, on the contrary, be shown that they prefer an orientation of the actin network towards the direction of zero strain instead of stress. ${ }^{48}$ This resulted in an orientation, on average, of $60^{\circ}$ to $70^{\circ}$ depending on the magnitude of the cyclic stretching.

\section{Conclusions}

We developed a microfluidic chamber that mimics the flow environment as found in larger human veins and simultaneously enables us to measure the contractile forces of human 
blood platelets. We characterized the spatio-temporal force development of blood platelets exposed to three different shear rates as well as without flow. For the comparatively low shear rates used here, the flow does not influence the contractile behavior in terms of spread area (about $40 \mu \mathrm{m}^{2}$ ), position of the force maxima, the maximum force magnitude (up to $500 \mathrm{nN}$ ) or the degree of anisotropy (about 2.2 on average). However, the contraction orientation is influenced. With increasing shear rate, the platelets move the direction of contraction away from the flow direction, until an angle of nearly $90^{\circ}$ is reached at the highest tested shear rate. This orientation proved to be stable from the time-point of attachment until the end of the recorded contraction. We thus conclude that the time-point of attachment determines the orientation of the cell during further spreading and contraction. We explain our observation using a physical model that accounts for the shear forces generated by the fluid flow, the resulting deformation of the platelets and the stresses being built up inside the cell volume and in particular along the circumference of the attached cell. Combining the simulation of the stresses with a simplified model of the contraction apparatus of the platelet, we deduced that with increased external force, the platelet aligns itself such that the stress acting on its adhesion sites is reduced. Such an alignment directly results in a more stable blood clot, decreasing the probability of detachment from the vessel wall. Our results indicate that this response to external forces is gradual and starts at very low physiological shear rates. Future experiments involving the current set-up and fluorescent stainings of the actin cytoskeleton ${ }^{45}$ or the usage of integrin force sensors $^{17}$ may help to experimentally verify the assumption of a correlation between the internal structures of the platelet and the force alignment. Our single cell study shows that clot stability is already initiated by the settlement of the very first platelet.

Due to the modular and flexible design of the microfluidic flow chamber, it is straight-forward to adapt the set-up for studying the influence of different drugs on the adhesion and contraction process. An adjustment of the shear rates with a corresponding change in the coating protein to collagen or von Willebrand factor would allow for studying physiological conditions comparable to smaller blood vessels. Additionally, the microfluidic setup could be used to study the build-up of blood clots while simultaneously measuring the corresponding contractile forces in future experiments.

\section{Conflicts of interest}

There are no conflicts to declare.

\section{Acknowledgements}

The authors acknowledge technical support by Aishwarya Paknikar and Tim Dullweber, as well as Assaf Zemel, Ulrich Schwarz and Dimitri Probst for many fruitful discussions. We thank Heidi Somsel for critical proofreading of the manuscript. This work was funded by the Deutsche Forschungsgemeinschaft (DFG) in the framework of the SFB 937, project A12.

\section{References}

1 A. D. Michelson, Platelets, Elsevier/Academic Press, Amsterdam and London, 2nd edn, 2007.

2 B. Diagouraga, A. Grichine, A. Fertin, J. Wang, S. Khochbin and K. Sadoul, J. Cell Biol., 2014, 204, 177-185.

3 S. Sorrentino, J.-D. Studt, O. Medalia and K. T. Sapra, Eur. J. Cell Biol., 2015, 94, 129-138.

4 D. Aquino, A. Schönle, C. Geisler, C. V. Middendorff, C. A. Wurm, Y. Okamura, T. Lang, S. W. Hell and A. Egner, Nat. Methods, 2011, 8, 353-359.

5 R. Sandmann, S. Schwarz Henriques, F. Rehfeldt and S. Köster, Soft Matter, 2014, 10, 2365-2371.

6 J. Hanke, D. Probst, A. Zemel, U. Schwarz and S. Köster, Soft Matter, 2018, 14, 6571-6581.

7 T. G. Papaioannou and C. Stefanadis, Hellenic J. Cardiol., 2005, 46, 9-15.

8 H. Hosseinzadegan and D. Tafti, Biotechnol. Bioeng., 2017, 114, 2154-2172.

9 B. Savage, E. Saldívar and Z. M. Ruggeri, Cell, 1996, 84, 289-297.

10 E. Gutierrez, B. G. Petrich, S. J. Shattil, M. H. Ginsberg, A. Groisman and A. Kasirer-Friede, Lab Chip, 2008, 8, 1486.

11 C. G. Conant, M. A. Schwartz, J. E. Beecher, R. C. Rudoff, C. Ionescu-Zanetti and J. T. Nevill, Biotechnol. Bioeng., 2011, 108, 2978-2987.

12 W. S. Nesbitt, S. Kulkarni, S. Giuliano, I. Goncalves, S. M. Dopheide, C. L. Yap, I. S. Harper, H. H. Salem and S. P. Jackson, J. Biol. Chem., 2001, 277, 2965-2972.

13 S. Feghhi, W. W. Tooley and N. J. Sniadecki, J. Biomech. Eng., 2016, 138, 104506.

14 X. M. Liang, S. J. Han, J.-A. Reems, D. Gao and N. J. Sniadecki, Lab Chip, 2010, 10, 991.

15 W. A. Lam, O. Chaudhuri, A. Crow, K. D. Webster, T.-D. Li, A. Kita, J. Huang and D. A. Fletcher, Nat. Mater., 2010, 10, 61-66.

16 S. Schwarz Henriques, R. Sandmann, A. Strate and S. Köster, J. Cell Sci., 2012, 125, 3914-3920.

17 Y. Wang, D. N. LeVine, M. Gannon, Y. Zhao, A. Sarkar, B. Hoch and X. Wang, Biosens. Bioelectron., 2018, 100, 192-200.

18 S. S. Hur, J. C. del Álamo, J. S. Park, Y.-S. Li, H. A. Nguyen, D. Teng, K.-C. Wang, L. Flores, B. Alonso-Latorre, J. C. Lasheras and S. Chien, Proc. Natl. Acad. Sci. U. S. A., 2012, 109, 11110-11115.

19 C. M. Perrault, A. Brugues, E. Bazellieres, P. Ricco, D. Lacroix and X. Trepat, Biophys. J., 2015, 109, 1533-1536.

20 P. Nghe, S. Boulineau, S. Gude, P. Recouvreuy, J. S. van Zon and S. J. Tans, PLoS One, 2013, 8, e75537.

21 D. R. Myers, Y. Qiu, M. E. Fay, M. Tennenbaum, D. Chester, J. Cuadrado, Y. Sakurai, J. Baek, R. Tran, J. C. Ciciliano, B. Ahn, R. G. Mannino, S. T. Bunting, C. Bennett, M. Briones, A. Fernandez-Nieves, M. L. Smith, A. C. Brown, T. Sulchek and W. A. Lam, Nat. Mater., 2016, 16, 230-235.

22 T. Das, T. K. Maiti and S. Chakraborty, Lab Chip, 2008, 8, 1308. 
23 R. H. W. Lam, Y. Sun, W. Chen and J. Fu, Lab Chip, 2012, 12, 1865-1873.

24 R. A. Jannat, M. Dembo and D. A. Hammer, Biophys. J., 2011, 101, 575-584.

25 L. Boldock, C. Wittkowske and C. M. Perrault, Microcirculation, 2017, 24, e12361.

26 R. Sinha, V. Nico, L. G. Séverine, A. van den Berg, B. Koopman and J. Rouwkema, Sci. Rep., 2016, 6, 2951.

27 R. Sandmann and S. Köster, Sci. Rep., 2016, 6, 22357.

28 D. C. Duffy, J. C. McDonald, O. J. A. Schueller and G. M. Whitesides, Anal. Chem., 1998, 70, 4974-4984.

29 J. C. McDonald and G. M. Whitesides, Acc. Chem. Res., 2002, 35, 491-499.

30 V. Leytin, D. J. Allen, E. Lyubimov and J. Freedman, Br. J. Haematol., 2007, 136, 762-764.

31 J. P. Califano and C. A. Reinhart-King, Cell. Mol. Bioeng., 2010, 3, 68-75.

32 H. Delanoë-Ayari, S. Iwaya, Y. T. Maeda, J. Inose, C. Rivière, M. Sano and J.-P. Rieu, Cell Motil. Cytoskeleton, 2008, 65, 314-331.

33 K. L. Hui, L. Balagopalan, L. E. Samelson and A. Upadhyaya, Mol. Biol. Cell, 2015, 26, 685-695.

34 Y. Aratyn-Schaus, P. W. Oakes and M. L. Gardel, Mol. Biol. Cell, 2011, 22, 1330-1339.

35 E. Kröner, Arch. Ration. Mech. Anal., 1959, 4, 273-334.

36 U. S. Schwarz and S. A. Safran, Phys. Rev. Lett., 2002, 88, 048102.
37 A. Zemel, F. Rehfeldt, A. E. X. Brown, D. E. Discher and S. A. Safran, J. Phys.: Condens. Matter, 2010, 22, 194110.

38 C. Hirsch, Numerical Computation of Internal and External Flows. The Fundamentals of Computational Fluid Dynamics, Elsevier, 2nd edn, 2007.

39 C. R. Harmison, R. H. Landaburu and W. H. Seegers, J. Biol. Chem., 1961, 236, 1693-1696.

40 Comsol Multiphysics, User's Guide CFD Module, 2013.

41 H. Mang and G. Hofstetter, Festigkeitslehre, Springer, Wien, 1st edn, 2000.

42 Y. Okamura, R. Schmidt, I. Raschke, M. Hintze, S. Takeoka, A. Egner and T. Lang, Biophys. J., 2011, 100, 1855-1863.

43 C. M. Edwards and U. S. Schwarz, Phys. Rev. Lett., 2011, 107, 128101.

44 A. Itakura, J. E. Aslan, S. Sinha, T. C. White-Adams, I. A. Patel, R. Meza-Romero, A. A. Vandenbark, G. G. Burrows, H. Offner and O. J. McCarty, J. Neuroinflammation, 2010, 7,75 .

45 A. K. Paknikar, B. Eltzner and S. Köster, Prog. Biophys. Mol. Biol., 2018, DOI: 10.1016/j.pbiomolbio.2018.05.001.

46 J. M. Brockman, A. T. Blanchard, V. P.-Y. Ma, W. D. Derricotte, Y. Zhang, M. E. Fay, W. A. Lam, F. A. Evangelista, A. L. Mattheyses and K. Salaita, Nat. Methods, 2018, 15, 115.

47 R. Kaunas, P. Nguyen, S. Usami and S. Chien, Proc. Natl. Acad. Sci. U. S. A., 2005, 102, 15895-15900.

48 U. Faust, N. Hampe, W. Rubner, N. Kirchgeßner, S. Safran, B. Hoffmann and R. Merkel, PLoS One, 2011, 6, e28963. 